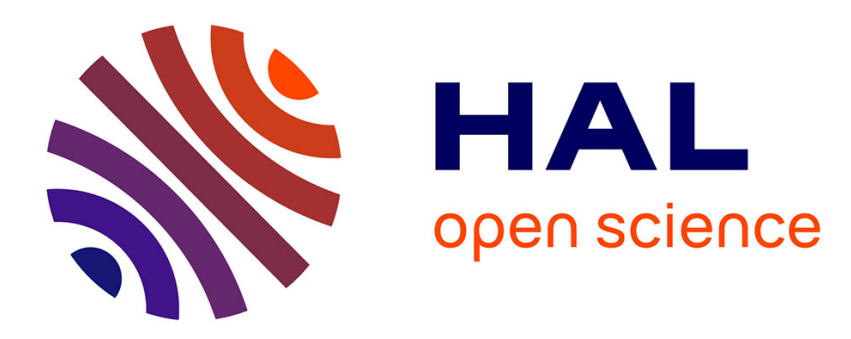

\title{
Modélisation de silencieux adaptés à la ventilation aéronautique
}

O. Planade, J. Guilhot

\section{To cite this version:}

O. Planade, J. Guilhot. Modélisation de silencieux adaptés à la ventilation aéronautique. Journal de Physique IV Proceedings, 1994, 04 (C5), pp.C5-105-C5-108. 10.1051/jp4:1994516 . jpa-00252839

\section{HAL Id: jpa-00252839 https://hal.science/jpa-00252839}

Submitted on 1 Jan 1994

HAL is a multi-disciplinary open access archive for the deposit and dissemination of scientific research documents, whether they are published or not. The documents may come from teaching and research institutions in France or abroad, or from public or private research centers.
L'archive ouverte pluridisciplinaire HAL, est destinée au dépôt et à la diffusion de documents scientifiques de niveau recherche, publiés ou non, émanant des établissements d'enseignement et de recherche français ou étrangers, des laboratoires publics ou privés. 


\title{
Modélisation de silencieux adaptés à la ventilation aéronautique
}

\author{
O. PLANADE et J.P. GUILHOT
}

L.A.M.I., 38 rue des 36 Ponts, 31000 Toulouse, France

\begin{abstract}
:
In aeronautic engineering, the ventilation noise is quite a problem for passager comfort. This study presents the acoustic waves propagation in acoustically lined tubes in the presence of mean flow. The aim of this work is to modelize the sound attenuation of reactive silencers in the case of circular or annular ducts.
\end{abstract}

\section{THÉORIE DES SILENCIEUX}

Le type de silencieux que nous sommes amenés à étudier ici, respecte les contraintes de poids et de dimension exigées dans le secteur aéronautique. Ainsi, afin de s'intéresser au comportement des ondes acoustiques dans ce dispositif, nous avons opté pour une approche modale du problème [1].

Afin de mieux exposer le phénomène théorique de propagation acoustique dans les conduits en présence d'écoulement d'air, nous émettons les hypothèses de travail suivantes :

- la section du conduit est circulaire

- la longueur du conduit est infinie

- le traitement acoustique est constitué d'un matériau absorbant muni ou non d'une tôle perforée, dont l'impédance acoustique Za est supposée constante sur toute la longueur du conduit

- le silencieux n'est soumis à aucun écoulement de fluide

Dans ces conditions, la solution de l'équation de propagation qui vérifie les conditions aux limites

s'écrit

$$
[p(r, \theta, z, t)]_{r \rightarrow 0}=p(0, \theta, z, t) \quad \text { et } \quad Z_{a}=\left[\frac{p(r, \theta, z, t)}{u(r, \theta, z, t)}\right]_{r \rightarrow a}
$$

$$
p(r, \theta, z, t)=\sum_{m, s} A_{(m, s) \cdot} J_{m}\left(k_{r,(m, s)} \cdot r\right) \cdot \cos (m \cdot \theta) \cdot \exp \left[\mathrm{j} \cdot\left(\omega \cdot t-k_{z,(m, s)} \cdot z\right)\right]
$$


Le nombre d'onde axial $\mathrm{kz}$ étant complexe, sa partie imaginaire $\gamma$ introduit un terme d'atténuation pour chaque mode $(m, s)$. Le calcul de $\gamma$ se fait en deux temps.:

- la résolution de la seconde condition aux limites, qui peut aussi s'écrire

$$
\eta \cdot J_{m}\left(k_{r,(m, s)} \cdot a\right)=k_{r,(m, s)} \cdot a \cdot J_{m}^{\prime}\left(k_{r,(m, s)} \cdot a\right) \quad \text { où } \quad \eta=j \cdot k \cdot a \cdot \frac{\rho \cdot c}{Z_{a}}
$$

où la solution correspond au nombre d'onde radial $\mathrm{kr}$ du mode considéré

- pour le même mode, le calcul de $\mathrm{kz}$ connaissant $\mathrm{kr}$ à l'aide de la relation de dispersion

$$
k^{2}=\left(\frac{\omega}{c}\right)^{2}=k_{z,(m, s)}^{2}+k_{r,(m, s)}^{2}
$$

Ainsi, nous pouvons en déduire l'atténuation modale $D_{(m, s)}(\mathrm{dB} / \mathrm{m})$.

L'atténuation globale du silencieux s'écrit alors :

$$
D=-20 \cdot \log \left[\sum_{(m, s)} A_{(m, s)} \cdot \exp \left(-\gamma_{(m, s)}, L\right)\right]
$$

où $A_{(m, s)}$ représente l'amplitude du mode $(m, s)$ et $L$, la longueur effective du silencieux.

En théorie, il existe une infinité de modes pour lesquels les équations précédentes sont vérifiées. Dans la réalité, il n'existe qu'un faible nombre de modes propagatifs, qui véhiculent l'énergie acoustique, et sur lesquels le calcul de l'atténuation est nécessaire.

Le calcul de l'amplitude modale implique la connaissance des sources acoustiques à l'entrée du silencieux, et l'impédance acoustique du matériau sur la gamme de fréquence considérée.

\section{MODÉLISATION DU SILENCIEUX}

La modélisation de ce type de dispositif se décompose en deux parties :

- Modélisation du traitement acoustique

- Modélisation de la propagation d'ondes acoustiques dans un silencieux

\subsection{Modélisation du traitement acoustique}

Cette modélisation repose sur les travaux de INGARD [2] concernant les matériaux poreux et les structures à panneaux perforés.

\subsection{1 Étude des matériaux}

L'impédance acoustique normale ramenée à l'avant du matériau poreux d'épaisseur $e$ est égale à : $Z_{\text {acoustique }}=-j Z_{c} \cot (\mathrm{ke})$

où $\mathrm{Zc}$ représente l'impédance caractéristique du matériau et $\mathrm{k}$, la constante de propagation dans ce matériau.

$$
\mathrm{Z}_{\mathrm{c}}=\rho \mathrm{c} \frac{\sqrt{\left[\mathrm{m}-\mathrm{j} \frac{\mathrm{r}}{\rho \omega}\right]}}{\sqrt{\mathrm{Pq}}} \quad \text { et } \quad k=\sqrt{P q} \frac{\omega}{c} \sqrt{\left[m-j \frac{r}{\rho \omega}\right]}
$$

Ces deux grandeurs s'écrivent comme fonctions des caractéristiques physiques du matériau :

- la porosité $P$ : définie comme étant le rapport du volume d'air contenu dans le matériau sur le volume total

- la densité $\rho$

- la résistance au passage de l'air $r$

- le facteur de structure $m$

Ces différents paramètres interviennent dans les équations de continuité et de la dynamique. Pour la plupart d'entre eux, leur détermination est réalisée expérimentalement. 


\subsubsection{Structure à panneaux perforés.}

Si on rajoute une tôle perforée d'épaisseur $h$ à l'avant du matériau, l'impédance acoustique normale se voit corrigée par deux termes de masse :

- un terme de masse dû au rayonnement de la face intérieure de la tôle perforée dans le matériau poreux : $+\mathrm{j} Z_{\mathrm{c}} \mathrm{k} \delta$

où $\delta$ correspond à la longueur corrigée de la tôle perforée.

- un terme de masse dû au rayonnement de la face extérieure de la tôle perforée dans l'air : $+\mathrm{jk} \mathrm{k}_{\mathrm{o}}(\mathrm{h}+\delta) \rho \mathrm{c}$

On obtient finalement l'impédance acoustique réduite par unité de surface :

$$
\mathrm{z}=\delta \frac{\mathrm{r}}{\rho \mathrm{c}} \frac{1}{\sigma}+\mathrm{j} \frac{1}{\sigma}\left[\mathrm{k}_{\mathrm{o}}\left(\mathrm{h}+(1+\mathrm{m}) \delta-\frac{\mathrm{z}_{\mathrm{c}}}{\rho \mathrm{c}} \cot (\mathrm{ke}) \cdot \sigma\right]\right.
$$

\subsection{Modélisation de la propagation d'ondes acoustiques dans un silencieux}

Sur les bases de la théorie des silencieux exposée précédemment, nous avons développé un logiciel de calcul prévisionnel de l'atténuation d'un silencieux de section circulaire ou annulaire, en présence d'écoulement.

Le point important de cette démarche réside dans la résolution de la deuxième condition aux limites. Nous pouvons montrer [3] que cette condition peut se ramener à une équation différentielle du premier ordre du type

$$
\frac{\mathrm{dy}}{\mathrm{d} \alpha}=\frac{-2 . \eta \cdot \mathrm{y}}{\mathrm{y}+(\eta \cdot \alpha)^{2}-\mathrm{m}^{2}} \quad \text { où } \quad y=\left(k_{r,(m, s)} \cdot a\right)^{2}
$$

La résolution de ce type d'équation, est effectuée à partir d'algorithme classique.

Connaissant le nombre d'onde radial, nous en déduisons le nombre d'onde axial, puis l'atténuation du mode considéré. La somme de la contribution de tous les modes propagatifs permet alors d'évaluer l'atténuation globale du silencieux.

Les difficultés rencontrées dans ce calcul portent d'une part sur l'étude en présence d'écoulement du comportement des matériaux utilisés, pour lesquels nous disposons de modèles théoriques, et d'autre part sur les conditions d'excitation d'un silencieux de dimension finie.

\section{MESURE DE L'ATTÉNUATION DU SILENCIEUX}

Des essais ont été réalisés sur un silencieux prototype. Le principe de la mesure de l'atténuation du silencieux consiste à relever la puissance acoustique en sortie du conduit, avec et sans le silencieux.

Soit $\mathrm{Lp}$ le niveau de puissance acoustique mesuré sans le silencieux, et Lw le niveau de puissance acoustique mesuré avec le silencieux, l'atténuation du silencieux est :

$$
D=L_{p}-L_{w}
$$

Plusieurs configurations expérimentales ont été définies selon les paramètres d'étude suivant :

- la densité du matériau absorbant.

- le taux de perforation de la tôle perforée.

- la présence effective ou non d'un bulbe placé sur l'axe du silencieux.

Les résultats expérimentaux sont représentés, fig.1 et 2 , avec les résultats obtenus à l'aide du modèle théorique, développé dans la deuxième partie.

\section{CONCLUSION}

Les premiers résultats de cette étude nous ont permis de valider notre modèle en absence d'écoulement. Les écarts observés entre les résultats théoriques et expérimentaux nous incitent à orienter la modélisation du silencieux sur des méthodes de type éléments finis, ceci afin de respecter les conditions réelles de propagation en présence d'écoulement. 


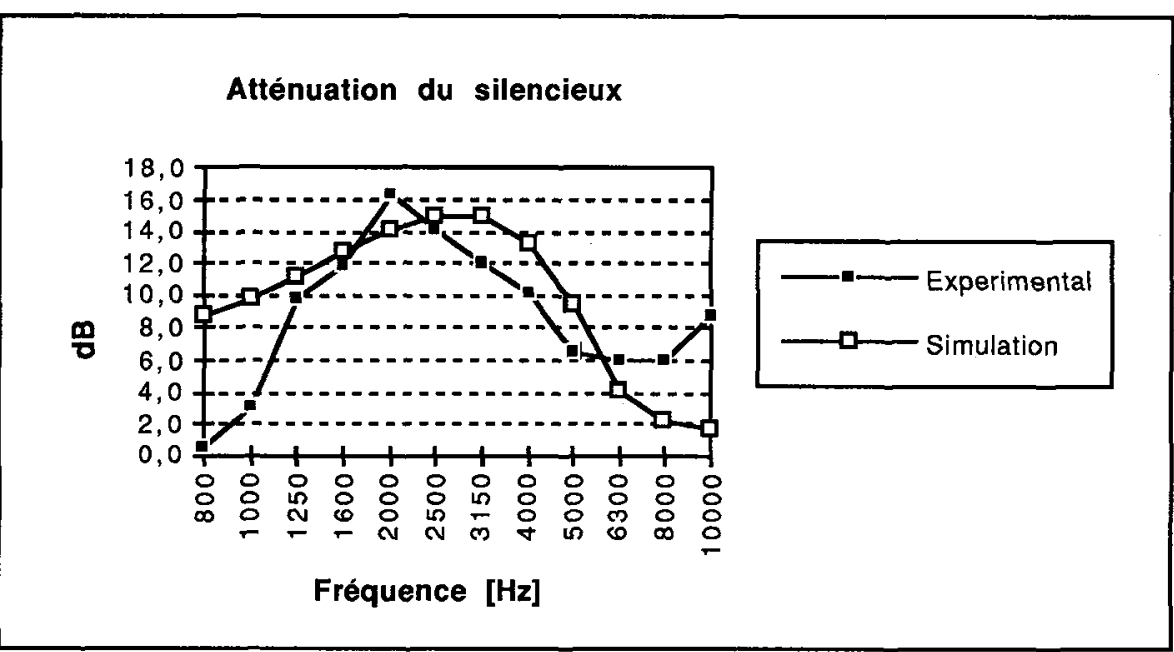

Fig. 1 : Comparaison théorie - expérimentation de l'atténuation du silencieux, en absence d'écoulement, dans la configuration matériau seul

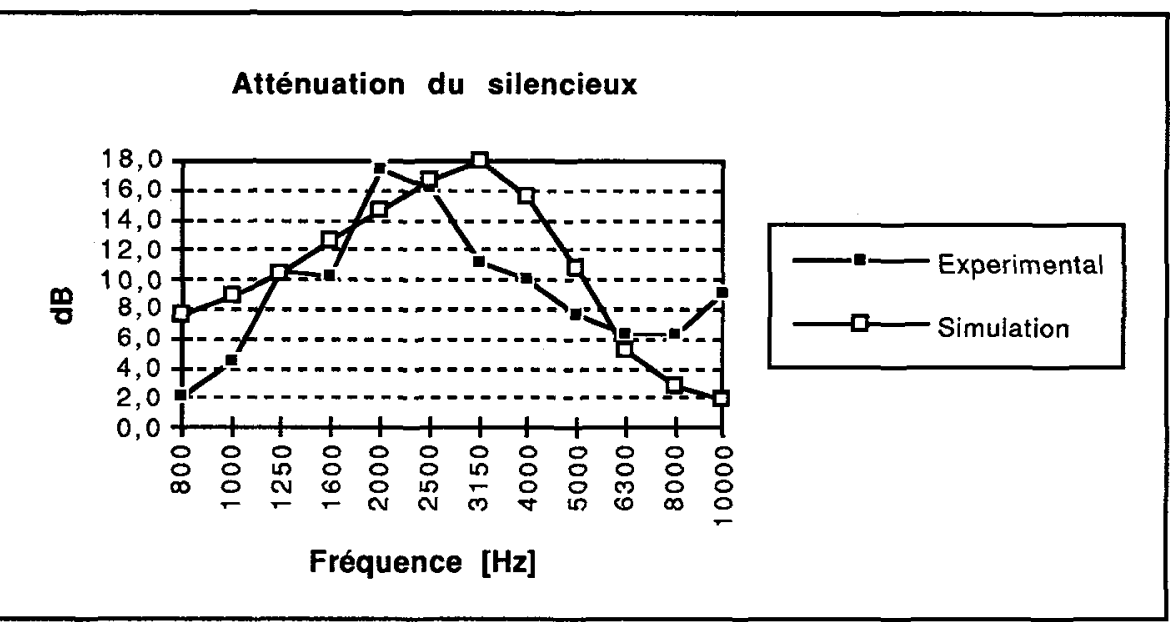

Fig.2 : Comparaison théorie - expérimentation de l'atténuation du silencieux, en absence d'écoulement, dans la configuration structure à panneaux perforés

\section{BIBLIOGRAPHIE}

[1] MORSE - INGARD, Theoretical Acoustics, Mc Graw Hill, 1968.

[2] INGARD U., Absorption characteristics of acoustic material with perforated facing, J.A.S.A., vol. $23 N^{\circ} 5$, Septembre 1947.

[3] EVERSMAN W., Computation of axial and transverse wave numbers for uniform twodimensionnal ducts with flow using numerical integration scheme, Journal of Sound Vibration, vol.41 N² p.252-255, 1975. 